\title{
Hypofractionated radiotherapy in low risk prostate cancer, preliminary experience
}

\begin{abstract}
Objective, Get free interval biochemical failure and toxicity in patients treated with hypofractionated radiotherapy (HFRT, for its acronym in English, Hypofractionated Radiation Therapy) with IMRT (Intensity Modulated Radiation Therapy), administering 70 Gy in 28 fractions of $2.5 \mathrm{~Gy}$.

Methods, In May 2013 began in CDD Unit of Radiotherapy and Radiosurgery of Abreu Clinic, an hypofractionation program with IMRT as an alternative treatment for patients with localized prostate cancer favorable risk. The four selected patients had histologically confirmed prostate adenocarcinoma without prior radical treatment and categorized as low risk according to the criteria of D'Amico et al. For each patient a treatment plan is made based on Intensity Modulated Radiation Therapy (IMRT) more Image Guided Radiation Therapy (IGRT) with $18 \mathrm{MV}$ photons from a linear accelerator CLINAC 21iX of the

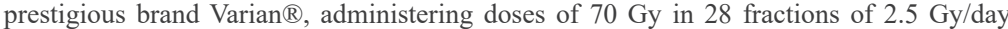
using an $\alpha / \beta 3$, biologically equivalent to 76 Gy in 38 fractions of $2 \mathrm{~Gy}$, under-Quadratic Linear Model. Patients were followed every 3 months for 18 months. The gastrointestinal and genitourinary toxicity $(\mathrm{GI})(\mathrm{GU})$, was evaluated prospectively and classified according to the criteria of the RTOG (Radiation Therapy Oncology Group). As a definition of biochemical failure we use the RTOG criteria (definition Pheonix), considered the elevation of post-radiotherapy $\mathrm{PSA} \geq 2 \mathrm{ng} / \mathrm{mL}$ above the nadir.
\end{abstract}

Results, During follow-up of 18 months, all patients (100\%) were free of biochemical failure. Only one patient presented grade 2 acute genitourinary toxicity, without having reported genitourinary or gastrointestinal late toxicity. This study is preliminary, with short follow and discreet patient population, however, is the first program and the first publication of hypofractionated treatment for prostate cancer radiotherapy in our country.

Conclusion, Hypofractionated Radiotherapy (HFRT) is a valuable treatment option for patients with favorable risk prostate cancer, with an excellent result of biochemical control and low toxicity.

Keywords: hypofractionation; IGRT
Volume 2 Issue 5 - 2015

\author{
Luis Moreno Sánchez, Nathalie González \\ Cazaño \\ Radiotherapy and Radiosurgery Unit Abreu Clinic, Dominican \\ Republic
}

Correspondence: Luis Moreno Sanchez, Radiation Oncologist, Nuclear Physician and Nathalie Gonzalez Cazano, Radiation Oncologist, Radiotherapy and Radiosurgery Unit, CDD Radioterapia, Clinica Abreu, Arzobispo Portes, \#805. Santo Domingo, Dominican Republic, Email radioncoluis@gmail.com, gonzalezac.nathalie@gmail.com

Received: April 09, 2015 | Published: May 25, 2015
Abbreviations: HFRT, hypofractionated radiotherapy; IGRT, image guided radiation therapy; GI, genitourinary toxicity; CT, computed tomography; IMRT, intensity modulated radiation therapy; PTV, planning target volume

\section{Introduction}

Prostate cancer ranks first among malignant solid tumors in males $(26 \%)$ and is the second leading cause of cancer deaths $(9 \%)$ in men in the US. It is estimated that $80-85 \%$ of cases are organ-confined. ${ }^{1}$ The main prognostic factors for developing risk groups for prostate cancer include PSA, Gleason score and clinical stage, classified according to the criteria of D'amico at Risk Low, Medium and High. ${ }^{2,3}$ Several randomized trials have shown that increasing the dose of external radiation therapy with standard fractionation improves biochemical control in patients with localized prostate cancer.

However, at the expense of prolonging the overall treatment time, which could be a burden on the limited resources in some of our patients and discomfort too. ${ }^{4,5}$ Hypofractionated Radiotherapy (HFRT) is an attractive option for the treatment of localized prostate cancer strategy. In contrast to most other tumors, prostate cancer seems to have an $\alpha / \beta$ ratio low and therefore could obtain an improvement in the therapeutic relationship, using a biological equivalent dose (BED, for its acronym in English, Biological Equivalent Dose) would use less total dose, but at a higher daily fractionation. Radiobiological addition benefit allows hypofractionated RT decrease total treatment time which may be reflected in less expensive treatments. The conventional fractionation dose recommended for patients with lowrisk prostate cancer is $>75 \mathrm{~Gy}$; with hypofractionation doses most used and most bibliographic support are recommended by Pollack et al. (70.2 Gy/26 fractions of $2.7 \mathrm{~Gy} /$ fraction) or of Kupelian et al., ${ }^{6}(70$ Gy/28 fractions of $2.5 \mathrm{~Gy} /$ fraction). ${ }^{3,5,7}$

\section{Material and methods}

In May 2013 a program of hypofractionation with Intensity Modulated Radiation Therapy (IMRT, for its acronym in English) more Image Guided Radiation Therapy (IGRT, for its acronym in English), began in CDD Unit of Radiotherapy and Radiosurgery of Abreu Clinic as an alternative treatment for patients with lowrisk prostate cancer. The four selected patients had histologically confirmed adenocarcinoma of the prostate, without radical treatment and categorized as low risk according to the criteria of D'Amico et al. Extension studies such as CT (computed tomography) of the pelvis with contrast and bone scan prior to initiation of treatment were included. 
For each patient a plan of intensity modulated radiation therapy (IMRT) plus guided radiotherapy (IGRT) with $18 \mathrm{MV}$ photon linear accelerator CLINAC $21 \mathrm{iX}$ of the prestigious commercial Varian ${ }^{\circledR}$. A dose of $70 \mathrm{~Gy}$ in 28 fractions was performed $2.5 \mathrm{~Gy} /$ day, - according Linear Quadratic Model - using an $\alpha / \beta 3$ Gy is biologically equivalent to $76 \mathrm{~Gy}$ in 38 fractions of $2 \mathrm{~Gy}$. All patients underwent CT simulation with cuts $5 \mathrm{~mm}$ supine with knees immobilizer, triangular foam support block and ankles, with proper bladder filling and emptying of the rectum. The prostate, seminal vesicles, rectum, bladder and penile bulb were delimited in all patients. The GTV (gross tumor volume for its acronym in English) represented the prostate and seminal vesicles. The CTV (clinical target volume for its acronym in English) included the prostate, seminal vesicles and a margin of $0.5 \mathrm{~cm}$ in all directions, meanwhile the PTV (planning target volume for its acronym in English) included the CTV $+0.5 \mathrm{~cm}$ margin in all directions except the posterior margin (rectum). The rectum was contoured uniformly from the anal margin to sigmoid colon. Daily verification images prior to treatment were performed. Patients were assessed at follow-up visit every 3 months for 18 months. At each visit, evaluated prospectively gastrointestinal and genitourinary toxicity (GI) (GU), classified according to the criteria of the RTOG (Radiation Therapy Oncology Group). Late toxicity was defined as any documented toxicity 90 days after the last treatment with radiation. In all checks will be performed on each patient's physical exam and PSA blood test, were questioned and always assisted by a radiation oncologist. As a definition of biochemical failure criterion used the RTOG (definition Phoenix), considered elevated PSA $\geq 2 \mathrm{ng} / \mathrm{mL}$ above the nadir PSA.

\section{Results}

From May to August 2013 selected four patients to be treated with hypofractionated RT. All had histologically confirmed adenocarcinoma of the prostate and classified as low risk according to the criteria of D'Amico and colleagues (Table 1). Importantly, the 4 patients indicated their intention to radical radiotherapy, totally ruling out other known treatment options (surgery, active surveillance, watchful waiting, androgenic blockade HI-FU).

Table I clinical characteristic of the patients

\begin{tabular}{lllll}
\hline Age & Gleason & Clinical Stage & First PSA & Risk \\
\hline 69 & 5 & TIb & $0.67 \mathrm{ng} / \mathrm{mL}$ & Low \\
77 & IHC (CK903-) & TIc & $25.4 \mathrm{ng} / \mathrm{mL}$ & Low \\
63 & 6 & TIa & $7.73 \mathrm{ng} / \mathrm{mL}$ & Low \\
74 & 6 & TIa & $3.05 \mathrm{ng} / \mathrm{mL}$ & Low \\
\hline
\end{tabular}

\section{Patient I}

Male 69 years, diagnosed with prostate ADC, Gleason $5(3+2)$ with $22 \%$ of positives fragments to disease (T1b), with initial PSA $0.67 \mathrm{ng} / \mathrm{mL}$, and after androgen hormone blockade based on Acetate Leuprolide (Lupron-Depot $22.5 \mathrm{mg}$ ), quarterly, administered in a single application by indicating their urological surgeon before radiotherapy. It was for technical IMRT dose previously described (70 Gy in 28 sessions of 2.5 Gy/day) from May 27 to July 28, 2013. It was hard then contact treatment, so he attended follow-up visit 6 months post-RT PSA $0.46 \mathrm{ng} / \mathrm{mL}$ and 12 months at $0.42 \mathrm{ng} / \mathrm{mL}$. In his latest follow-up January 2015 (18 months) showed PSA of $0.39 \mathrm{ng} / \mathrm{mL}$, the patient was asymptomatic to date.

\section{Patient 2}

Male 77 years with localized prostate ADC, confirmed by immunohistochemistry (CK903 negative), with initial PSA $25.4 \mathrm{ng} /$
$\mathrm{mL}$. A scan Uro (CTU) reported enlarged $(60 \mathrm{~mm} \times 50 \mathrm{~mm})$ irregular contours and weighing approximately 62 grams prostate. By indicating their urological surgeon remained low androgen blockade (bicalutamide $150 \mathrm{mg}$ ) for 30 days before starting treatment with radiotherapy. She tolerated the treatment without complications from June 24 to August 7, 2013, receiving the same technique and aforementioned doses. At 3 months after completed treatment he attended the first consultation meeting completely asymptomatic Control and PSA $1.28 \mathrm{ng} / \mathrm{mL}$. At 6 months remained equally and PSA $0.94 \mathrm{ng} / \mathrm{mL}$, likewise at 12 months with PSA $0.82 \mathrm{ng} / \mathrm{mL}$. He attended the clinic for follow up of 18 months to January 2015, with PSA at $0.76 \mathrm{ng} / \mathrm{mL}$, uneventful.

\section{Patient 3}

Male 63 years with ADC prostate, Gleason 6 (3 3), with just $5 \%$ of positive biopsy fragments to tumor (T1a), with an initial PSA of 7.73 $\mathrm{ng} / \mathrm{mL}$. A transrectal prostate ultrasound (US) reported an enlarged prostate, with dimensions of $4.4 \times 4.7 \times 2.8 \mathrm{~cm}$, with approximately 31 grams. It remained under androgen deprivation for 2 months prior to treatment with RT in order of urological surgeon, based on bicalutamide $150 \mathrm{mg}$ once daily, orally. Treated like previous patients, from June 25 until August 13, 2013. Tolerated treatment satisfactorily, presenting mild to moderate dysuria gave analgesic use. At 3 months after completed treatment he attended follow-up visit with PSA 0.26 $\mathrm{ng} / \mathrm{mL}$, without any symptoms. At 6 months, went asymptomatic with PSA $0.29 \mathrm{ng} / \mathrm{mL}$. In his third visit at 9 months the PSA was at $0.43 \mathrm{ng} / \mathrm{mL}$; at 12 months the PSA was $0.22 \mathrm{ng} / \mathrm{mL}$. In his last visit at 18 months post-treatment PSA was found in $0.215 \mathrm{ng} / \mathrm{mL}$, even asymptomatic.

\section{Patient 4}

Male 74 years, diagnosed with prostate ADC, Gleason $6(3+3)$ with only 01 positive fragment for 10 biopsied disease $(<5 \%$, T1a) with initial PSA of $3.05 \mathrm{ng} / \mathrm{mL}$. CT of the pelvis with contrast reported, subtly enlarged prostate heterogeneous. It remained under androgen blockade by prior treatment with RT month, indicating their urological surgeon. It was from June 21 to August 9, 2013, with the same technique and dosages others. In his first follow-up at 3 months remained asymptomatic with PSA $0.77 \mathrm{ng} / \mathrm{mL}$. At 6 months, the PSA had dropped to $0.41 \mathrm{ng} / \mathrm{mL}$ and remained asymptomatic. In consultation control the PSA 9 months reported values of $0.17 \mathrm{ng} / \mathrm{mL}$, at 12 months $0.11 \mathrm{ng} / \mathrm{mL}$ and is currently at $0.09 \mathrm{ng} / \mathrm{mL}$ (18 months), Remained asymptomatic.

The acute toxicity was evaluated weekly during treatment and out as required by the patient. All patients tolerated the treatment without severe side effects except one patient reported mild dysuria improved with the use of common antispasmodics (Grade 2). After the first 3 months after RT, no patient had gastrointestinal or genitourinary symptoms, the patient had presented dysuria reported at their first post-RT controlling the discomfort was gone. In general, patients had no symptoms GU or GI for 3 months after completion of treatment. Late toxicity was assessed every 3 months after the first consultation control after radiotherapy, where the four patient's refierieron be asymptomatic, leading a normal life.

\section{Biochemical control}

During follow up of 18 months, our four (04) patients remain free from biochemical failure, PSA within normal limits, with no increase representing a risk, all with values $<1 \mathrm{ng} / \mathrm{mL}$ (Figure 1). This study is still preliminary, with short follow and discreet patient 
population, however, is the first program and the first publication of hypofractionated treatment for prostate cancer radiotherapy in our country.
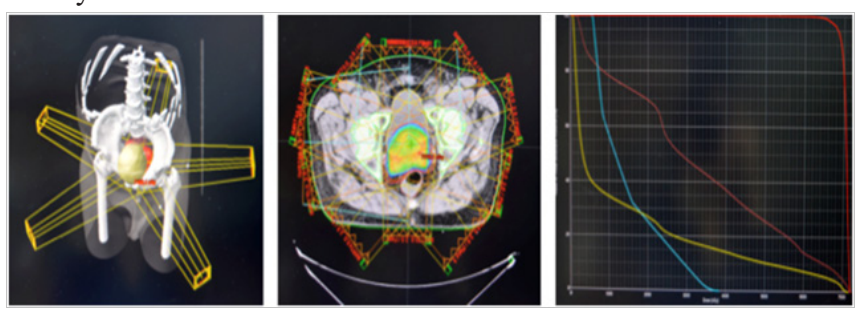

Figure I Planning HFRT with IMRT.

\section{Discussion}

In recent years, there has been increasing interest in evaluating the effect of dose per fraction in prostate cancer. Hypofractionated radiotherapy (HFRT) is a method that optimizes the fractions of radiotherapy, designed to increase daily fraction dose by delivering total dose in a shorter period of time. Several clinical data have suggested that the radiotherapeutic treatment of prostate cancer improves with hypofractionation (higher dose per fraction with an abbreviated general course of radiotherapy). ${ }^{8,9}$ The conventional fractionation scheme employing 1.8-2.0 Gy fractions is based on the premise that tumors are usually less sensitive to dose/daily low relative to normal tissues delayed response. The $\alpha / \beta$ ratio is a measure of the response fractionation low proportions associated with normal tissues that respond later. The unusual aspect of this radiobiology relates to high atypical prostate cancer sensitivity to high daily fractions of radiation.

The answer fractionation ( $\alpha$ ratio/ $\beta$ ) of prostate cancer and normal tissues of late response, not yet defined rigorously. Additionally, the linear model quadratic (LQ) used in designing treatments hypofractionated RT is subject to its own uncertainties, particularly with respect to the upper limit for the dose per fraction which remains valid. There are exceptions to that tumor response to typical fractionation, however, the growth fraction (effective time of the cell cycle) has often been associated with response to fractionation, with normal proliferating tissues slowly (and some tumors that grow slowly) to generally exhibit a response majority expected a higher dose per fraction (low $\alpha / \beta$ ratio). This relationship has been shown for melanoma, some types of sarcomas, and prostate cancer. ${ }^{9}$

In principle, the use of hypofractionation was motivated by concerns of cost and availability of resources, without relating to the expectation of a biological advantage. Unlike other epithelial tumors it has been speculated that the $\alpha / \beta$ ratio of prostate cancer is less than that of the surrounding normal tissues. An $\alpha / \beta$ ratio means more low tumor sensitivity to the magnitude of the fraction, and therefore an increase in dose per fraction over 1.8 or 2.0 Gy (conventional fractionation) provides a therapeutic benefit. ${ }^{3,10}$ The first suggestion that the $\alpha / \beta$ ratio for prostate cancer is about $1.5 \mathrm{~Gy}$ was obtained from an analysis of the results by comparing patients with permanent seed implant brachytherapy dose of $145 \mathrm{~Gy}$ and another group treated with external beam radiotherapy (RTE) and conventional fractionation dose of 70-74 Gy. Fowler \& colleagues calculated a similar estimate of $\alpha / \beta$ ratio of 2 Gy with men treated by RTE and brachytherapy with iodine-125 (I125) or Palladium-103 (Pd103). Brenner and colleagues completed another estimate of $\alpha / \beta$ ratio with a group of patients treated at a dose escalation trial using high dose rate brachytherapy (HDR, for its acronym in english) with doses per fraction from 5.5 to $10.5 \mathrm{~Gy}$.
Patients treated with HDR brachytherapy had a better biochemical control with an $\alpha / \beta$ ratio of $1.2 \mathrm{~Gy}$. In a series of 3,756 patients treated with external beam radiotherapy, which included 185 patients after RTE received an overlay or "boost" with HDR brachytherapy, Williams and colleagues calculated an $\alpha / \beta$ ratio of $2.6 \mathrm{~Gy}$. In general, it is believed that the $\alpha / \beta$ ratio for the majority of cancers is as high as $10 \mathrm{~Gy}$, but for prostate cancer have been suggested values about 1.5 Gy, which is less than 3 Gy indicated for most normal tissue.,, 10

In our hypofractionated radiotherapy (HFRT) program, we use the same scheme Kupelian et al., ${ }^{6}$ (70 Gy in 28 fractions of 2.5 Gy), one of the most widely used internationally, with high biological equivalent dose (BED, for its acronym in English) and more bibliographic importance, being equivalent to 76 radiobiológicamente Gy in 38 fractions 2 Gy, with $\alpha / \beta 3$ Gy (Table 2 ) relationship. There are several obvious advantages for fractionated treatment, including patient comfort because it offers significantly fewer visits, and lower cost. Various studies have been conducted to evaluate the effect of HFRT compared with conventional fractionation radiotherapy (CFRT, for its acronym in English, Fractionationed Conventionally Radiation Therapy) have reported similar oncologic outcomes; even some have reported promising results in control of biochemical failure with hypofractionated radiotherapy. It has been shown that high radiation doses per fraction is higher than the conventional dose in preventing biochemical failure in patients with prostate cancer; biochemical failure rate is significantly lower in the group HFRT, with an absolute benefit of $30 \%$, but has not reported significant difference in mortality between HFRT and CFTR. ${ }^{4}$ Hypofractionated treatments with higher doses per fraction, theoretically produce more toxicity while providing greater therapeutic benefit in controlling tumor. However, Arcangeli et al., ${ }^{9}$ suggested that the hypofractionated scheme leads only to a slight and non-significant increase in acute toxicity and temporal tolerable, while the severity and frequency of late complications is equivalent to those observed in conventional treatment. ${ }^{4,5,11}$ In our study, only one (01) patients reported genitourinary toxicity early in the third week of treatment; none developed acute gastrointestinal toxicity and to date, with 18 months of follow up, all patients are asymptomatic.

Table 2 Biological effective dose (BED) assuming an $\alpha / \beta$ of $3 G y$ for different schemes HFRT

\begin{tabular}{ll}
\hline Author & BED3 \\
\hline Lukka et al. & 98 \\
Zietman et al. ${ }^{23}$ (standard dose) & 112 \\
Kuban et al..$^{24}$ (standard dose) & 116 \\
Martin et al & 120 \\
Zelefsky et al & $>121$ \\
Dearmaley et al & 123 \\
Zietman et al. ${ }^{23}$ & 126 \\
Kupelian et al. ${ }^{6}$ & 128 \\
Kuban et al..$^{24}$ (escalated dose) & 130 \\
McGill University & 132 \\
Akimoto et al & 138 \\
\hline
\end{tabular}

\section{BED, biologically effective dose}

Moreover, androgen deprivation therapy has not shown to have any effect on local control and free time to biochemical failure in patients treated with HFRT. ${ }^{5,10,12}$ The hormonal association with radiotherapy has several objectives, reducing prostate volume, leading to a volume reduction treatment and thus an improvement of dose-volume histograms (HDV); reduce the risk of local relapse in the volume irradiated to inhibit cell recruitment during irradiation; 
reducing metastases due to the presence of subclinical tumor deposits distance and improve the effectiveness of radiotherapy or supraadditive through additive effect. Regardless, in the group of patients with low risk, the association of hormonal treatment is indicated only in highly selected patients with a large prostate volume would be used as neoadjuvant therapy in order to reduce prostate volume. ${ }^{2}$ All patients selected for our program hypofractionation received neoadjuvant hormone androgen blockade with one to three months indicating their urological surgeon, either by prostate volume large or because the patient had not yet decided the treatment modality that would receive and his urologist wanted to avoid progressive elevation of PSA.

Analysis in a Cleveland Clinic HFRT produced a $82 \%$ control of biochemical failure to 5 years, relatively equivalent or better than previously achieved with standard institutional 78 Gy at 2 Gy per fraction. Other studies have determined a general biochemical control rate of $83 \%$ at 5 years. Our patients, with one year follow up, are low PSA levels, none has achieved an increase that meets the established class definition for biochemical failure in Phoenix; to date our control is $100 \%$, considering it is a study program at a single institution hypofractionation reporting preliminary results. ${ }^{13-19}$

\section{Conclusion}

Hypofractionated radiotherapy (HFRT) is a valuable treatment option for patients with good-risk localized prostate cancer, with an excellent result of biochemical control and low toxicity. Compared with conventional fractionation radiotherapy (CFRT) some studies have reported benefits in the long term biochemical control with similar toxicity. The most used and most bibliographic support doses are recommended by Pollack et al. (70.2Gy/26 fractions of $2.7 \mathrm{~Gy} /$ fraction) or of Kupelian et al., ${ }^{6}$ (70Gy/28 fractions of $2.5 \mathrm{~Gy} /$ fraction). ${ }^{20-22}$ Moreover, treatment with androgen deprivation has not shown to have any effect on local control and free time to biochemical failure in patients treated with HFR. Other prospective research is needed to explore the optimal daily fraction, including a protocol margins planning volume (PTV), management techniques, dose and oncological results of longer periods of follow-up..$^{23,24}$

\section{Acknowledgments}

None.

\section{Conflicts of interest}

The author declares that there is no conflict of interest.

\section{Funding}

None.

\section{References}

1. Siegel R, Ma J, Zou Z, et al. Cancer Statistics. CA Cancer J Clin. 2014;64(1):9-29.

2. Leonard L, Gunderson MD, Jel E, et al. Clinical Radiation Oncology. $3^{\text {rd }}$ ed. Elsevier, Canada; 2012.

3. Sun L, Zhu S, Zhao Y, et al. Who benefits from hypofractionated radiationtherapy for clinically localized prostate cancer: evidence from meta-analysis. Tumor Biol. 2014;35(10):9911-9918.

4. Yeoh EE, Botten RJ, Butters J, et al. Hypofracionated Versus Conventionally Fractionated Radiotherapy for Prostate Carcinoma: Final Results Of Phase IIIRandomized Trial. Int J Radiat Oncol Biol Phys. 2011;81(5):1271-1278.
5. Lopez MA, AlpuenteLA, BadalIranzo MD, et al. Manual Prácticode OncologíaRadioterápica SEOR. 2013.

6. Kupelian PA, Reddy CA, Carlson TP, et al. Preliminary observations on biochemical relapse-free survival rates after short-course intensitymodulated radiotherapy ( $70 \mathrm{~Gy}$ at $2.5 \mathrm{~Gy} /$ fraction) for localized prostate cancer. Int J Radiat Oncol Biol Phys. 2002;53(4):904-912.

7. Nita Patel, Sergio Faria, Fabio Cury, et al. Hypofractionated Radiation Therapy ( 66 Gy in 22 Fractions at 3 Gy per Fraction) for Favorable-Risk Prostate Cancer:Long-term Outcomes. IntJ Radiat Oncol Biol Phys. 2013;86(3):534-539.

8. Mark Ritter Rationale, conduct, and outcome using hypofractionated radiotherapy in prostate cancer. Semin Radiat Oncol. 2008;18(4):249256.

9. Arcangeli S, Strigari L, Gomellini S, et al. Updated results and patterns of failure in a randomized hypofractionation trial for high-risk prostate cancer. Int J Radiat Oncol Biol Phys. 2012;84(5):1172-1178.

10. ArrudaViani G, Stefano EJ, Alfonso SL Higher than conventional radiation doses in localized prostate cancer treatment:A meta-analysis of randomized, controlled trials. Int J Radiat Oncol Biol Phys. 2009;74(5):1405-1418.

11. Livsey JE,Cowan RA,Wylie JP, et al. Hypofractionated Conformal Radiotherapy in Carcinoma of the Prostate:five-year Outcome Analysis. Int J Radiat Oncol Biol Phys. 2003;57(5):1254-1259.

12. Roach MR, Hanks G, Thames HJ, et al. Defining biochemical failure following radiotherapy with or without hormonal therapy in men with clinically localized prostate cancer:recommendations of the RTOGASTRO Phoenix Consensus Conference. Int J Radiat Oncol Biol Phys. 2006;65(4):965-974.

13. Dearnaley D, Syndikus I, Sumo G, et al. Conventional versus hypofractionated high-dose intensity modulated radiotherapy for prostate cancer:preliminary safety results from the CHHiPrandomised controlled trial. Lancet Oncol. 2012;13(1):43-54.

14. Cho LC, Timmerman R, Kavanagh B Hypofractionated External-Beam Radiotherapy for Prostate Cancer. Prostate Cancer. 2013;2013:103547.

15. Fowler JF Emeritus of Medical School of Wisconsin University, Department of Human Oncology, University of Wisconsin-Madison, USA, Acta Oncologica. 2005;44:265-276.

16. Leborgne F, Fowler J, Leborgne JH, et al. Later outcomes and alpha/ beta estimate from hypofractionated conformal three-dimensional radiotherapy versus standard fractionation for localized prostate cancer. Int J Radiat Oncol Biol Phys. 2012;82(3):1200-1207.

17. Di Staso M, Bonfili P, Gravina GL, et al. Late morbidity and oncological outcome after radical hypofractionated radiotherapy in men with prostate cancer. BJU Int. 2010;106(10):1458-1462.

18. Mok G, Martin J, Massey C, et al. A comparison of conventional and hypofractionated radiotherapy schedules in the treatment of localized prostate cancer. Int J Radiat Oncol Biol Phys. 2011;81:S401-S402.

19. Brenner DJ, Martinez AA, Edmundson GK, et al. Direct evidence that prostate tumors show high sensitivity to fractionation (low alpha/beta ratio) similar to late-responding normal tissue. Int J Radiat Oncol Biol Phys. 2002;52(1):6-13.

20. Fowler JF, Ritter MA, Chappell RJ, et al. What hypofractionated protocols should be tested for prostate cancer? Int J Radiat Oncol Biol Phys. 2003;56(4):1093-1104.

21. Sethukavalan P, Cheung P, Tang CI, et al. Patient costs associated with external beam radiotherapy treatment for localized prostate cancer:the benefits of hypofractionated over conventionally fractionated radiotherapy. Can J Urol. 2012;19(2):6165-6169. 
22. Miralbell R, Roberts SA, Zubizarreta E, et al. Dose-fractionation sensitivity of prostate cancer deduced from radiotherapy outcomes of 5,969 patients in seven international institutional datasets: alpha/beta =1.4 (0.9-2.2) Gy. Int J Radiat Oncol Biol Phys. 2012;82(1):e17-e24.

23. Zietman A, DeSilvio M, Slater J, et al. Comparison of Conventional-
Dose vs High-Dose Conformal Radiation Therapy in Clinically Localized Adenocarcinoma of the Prostate:A Randomized Controlled Trial. JAMA. 2005;294(10):1233-1239.

24. Kuban DA, Tucker SL, Dong L, et al. Long-termresults of the M.D. Anderson randomizeddose-escalation trialforprostatecancer. Int $J$ Radiat Oncol Biol Phys. 2008;70(1):67-74. 